\title{
Electro-exfoliating graphene from graphite for direct fabrication of supercapacitor
}

\begin{abstract}
A facile production of graphene via electro-exfoliation is demonstrated using different types of oxidizing agent $\left(\mathrm{HNO}_{3}, \mathrm{NaNO}_{3}, \mathrm{H}_{2} \mathrm{SO}_{4}\right.$ and $\left.\mathrm{H}_{2} \mathrm{O}_{2}\right)$ in the presence of sodium dodecylbenzenesulfonate as a surfactant. Different types of surfactant-oxidizing agent solutions in different concentrations significantly influenced the electrochemical exfoliation of graphite rod. The surface morphology, layer thickness and defects of the as-produced graphene are further evaluated. Additionally, the as-produced graphene is fabricated as a supercapacitor electrode via direct vacuum filtration. Nylon membrane and polymer gel, each containing $2.0 \mathrm{M}$ of potassium hydroxide, are utilized to investigate the influence of the electrolyte type on the capacitance performance. Upon 1000 charge/discharge cycles, the nylon membrane electrolyte recorded capacitance retention of $94 \%$, whereas the polymer gel electrolyte recorded an impressive capacitance retention that exceeded $100 \%$. The potential of the fabricated supercapacitor for real applications is manifested by its ability to light up a light-emitting diode upon charging.
\end{abstract}

Keyword: Graphene; Surfactant; Oxidizing agent; Electrochemical exfoliation; Supercapacitor 